\title{
Experimental field research on nitrate balance in agricultural soils
}

\author{
A. M. Marinov ${ }^{1}$, M. Pele ${ }^{2}$, E. M. Draghici ${ }^{3}$, G. Vasile $^{4}$ \\ \& M. Artimon ${ }^{2}$ \\ ${ }^{I}$ Department of Hydraulics, Hydraulic Machinery and Environmental \\ Engineering, Faculty of Power Engineering, \\ University Politehnica of Bucharest, Romania \\ ${ }^{2}$ Chemistry Department, University of Agronomic Sciences and \\ Veterinary Medicine Bucharest (UASVMB), Romania \\ ${ }^{3}$ Vegetable Crops Department, (UASVMB), Romania \\ ${ }^{4}$ Agrochemistry Department, (UASVMB), Romania
}

\begin{abstract}
High nitrate levels in groundwater endanger public water supplies. Although the European Community sets $50 \mathrm{mg} \mathrm{NO}_{3}^{-} / \mathrm{L}$ as the maximum tolerated concentration in drinking water, this level is exceeded in many aquifers. The input concentrations of nitrates into the groundwater have to be reduced to acceptable levels. One of the measures to facilitate this reduction is the application of fertilizers according to the actual demand of plants and the existing quantity of nitrogen in the root zone prior to the application. Our work focuses on reducing soil contamination due to agricultural practices by analysis of irrigation and drainage with respect to transport of nitrogenous fertilizer through the soil. This paper describes our experimental field research developed in tunnels during the plants' (Lactuca Sativa L) life time. The concentration of nitrogen compounds in soil and the nitrogen uptake by plants were measured and compared for different fertilization schedules. We compare existing methods of fertilizer application, water management, and agricultural practices to improve nitrate pollution control in soils and we propose corresponding best management practices.
\end{abstract}

Keywords: soil pollution with nitrate, fertilization, irrigation, agricultural practices, experimental research. 


\section{Introduction}

A fundamental goal of subsurface water management is the protection of soil, water and groundwater resources against degradation and contamination. The natural capacity of aquifers to purify subsurface water must not be destroyed by contamination from industrial, agricultural, and domestic sources. Subsurface processes are extraordinarily slow, and toxic effects may only become evident several years or decades after a contaminant release. Rehabilitation of contaminated soils or aquifers requires many years, or even generations. Crop responses to nitrogen $(\mathrm{N})$ fertilizer are impressive and rewarding to farmers, leading them to exaggerate application rates. The over fertilization and over watering that are associated with irrigated row-cropping increase the probability of developing groundwater pollution problems (Pereira and Quelhas dos Santos [4]).

Although nitrogen fertilizers take several chemical forms, processes occurring in the soil lead to their transformation into nitrate-nitrogen $\left(\mathrm{N}-\mathrm{NO}_{3}{ }^{-}\right)$. Nitrates are highly soluble in soil water and are not retained by the chemical complex in the soil. Soil microorganisms retain $\mathrm{N}-\mathrm{NO}_{3}^{-}$only temporarily, and mineralization occurs later with the return of $\mathrm{N}$ to nitrate form. Mobile nutrients such as nitrogen in nitrate form move easily with water flow to deeper soil layers and to the groundwater.

Our work focuses on reducing soil contamination due to agricultural practices by analysis of (a) plant growth and (b) irrigation and drainage with respect to the transport of nitrogenous fertilizer through the soil and toward the plants' roots. For a tunnel row-crop of lettuce (Lactuca Sativa L) we developed an experimental design aimed at analyzing the fertilization influence on the soil pollution with nitrogenous compounds and on the plant growth. We measured the physical soil properties, nitrogen concentration in the soil profile and the plant nitrate content throughout the lettuce lifetime. The acquired data will be used in a future study to calibrate and improve a mathematical model (Marinov and Diminescu [3]), for subsurface pollution forecasting.

\section{Monitoring nitrogen migration in a field experiment}

The transport, storage, exchange and transformation processes of heat and solutions in the soil and groundwater zone are called migration processes. These processes are physically, chemically and biologically influenced by the soil, water, plants and pollutants properties.

Our research analyses the nitrogen migration process in six plant growth scenarios developed in an agricultural tunnel. Fig. 1 shows the lettuce field after three weeks of life. The goal of our research is to determine the influence of the fertilizer application rate and the influence of covering the field with plastic materials (mulch) on the nitrogen transfer in soil and plants during the lettuce's lifetime.

Usually the mulch method conserves up to $80 \%$ of the soil's moisture allowing irrigation water to be more efficiently used; significantly moderates soil temperature fluctuations; improves soil health, and plant productivity; avoids soil 


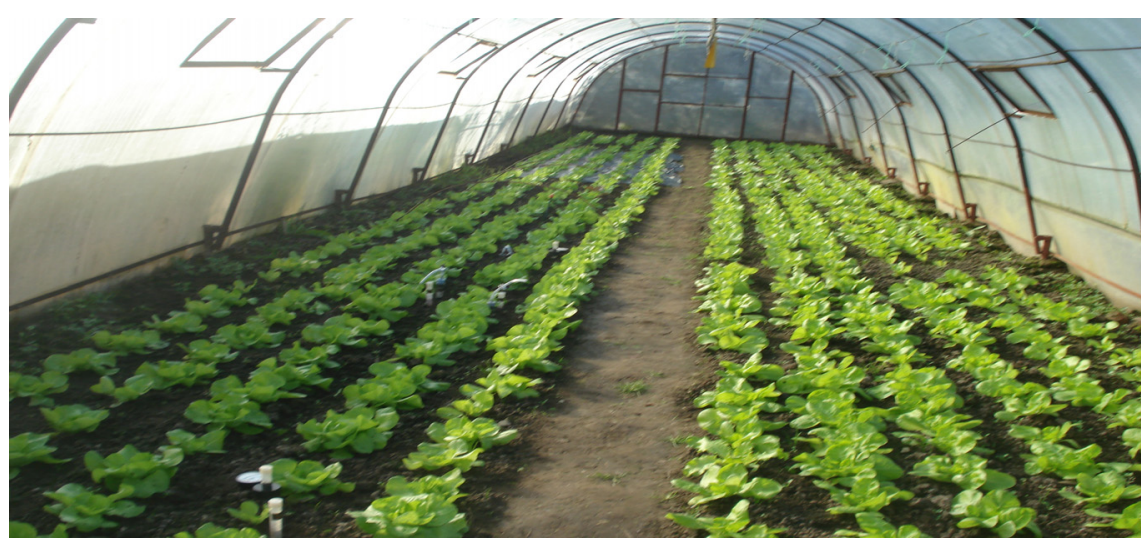

Figure 1: $\quad$ Lettuce field in the third week of plant life.

erosion problems; reduces or eliminates herbicide use; permits more efficient fertilizer use, with less leaching, and protects groundwater quality (Draghici [2]).

Initial soil measurements included grain-size distribution curve, field capacity, bulk density, soil specific gravity, and the volumetric water content at saturation.

Air and soil temperature $\left[{ }^{\circ} \mathrm{C}\right]$ and light intensity [klux] in the tunnel were measured daily (at 9 am and $2 \mathrm{pm}$ ), and the average daytime values are shown in fig. 2.
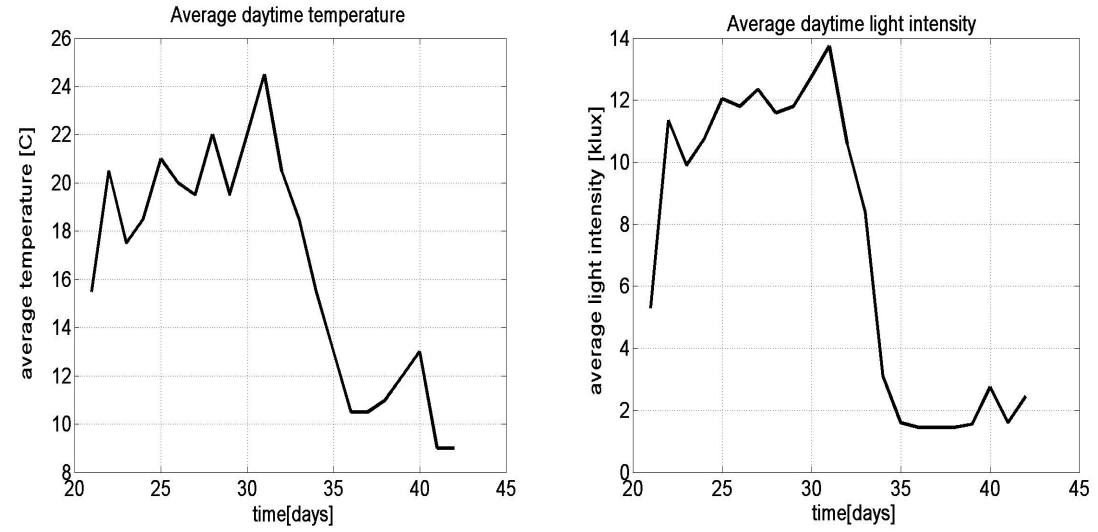

Figure 2: $\quad$ Average daytime values for air temperature and light intensity.

The following parameters were measured weekly during plant lifetime in six (F1-F6) separate subfields shown in fig. 3:

- for the soil profile, at different depths: volumetric water content, water pressure (suction), nitrogen concentration;

- for the plants: nitrogen concentration in leaves, nitrogen concentration in roots, plant growth parameters (leaf count, size and weight; plant diameter, height and volume, length of roots). 


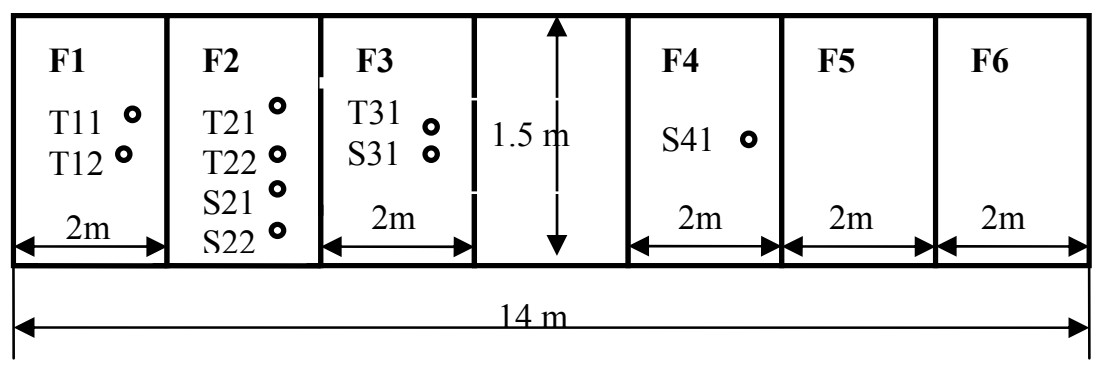

Figure 3: Field description and sampling instruments position. T11, T12, T21, T22, T31 are tensiometric tubes, S21, S22, S31, S41 are extraction tubes SPS200 (soil water sampling).

Each field has a $3 \mathrm{~m}^{2}$ surface and $5 * 9$ lettuces planted on 29.10.2009 (day 1). The fertilizer (Ammonium nitrate: $17 \% \mathrm{~N}-\mathrm{NO}_{3}{ }^{-}$and $17 \% \mathrm{~N}-\mathrm{NH}_{4}+$ ) dissolved in water was applied with the irrigation water over each field in the fourth week of plant lifetime (24.11.2009-day 26). A second fertilization identical with the first one was applied ten days later on 04.12.2009 (day 36). Fertilizer concentrations and soil covering procedures are detailed in table 1 .

Table 1: $\quad$ Experimental design.

\begin{tabular}{|c|c|c|c|c|c|c|}
\hline Field nr. & Field & $\begin{array}{c}\text { Irrigation } \\
\text { symbol }\end{array}$ & $\begin{array}{c}\text { Fertilizer } \\
\text { conc. } / \mathrm{m}^{2}\end{array}$ & $\mathrm{~N}-\mathrm{NO}_{3}$ & $\mathrm{~N}-\mathrm{NH}_{4}$ & $\begin{array}{c}\text { Covering } \\
\text { procedure }\end{array}$ \\
\cline { 3 - 7 } & & $\mathrm{L} / \mathrm{m}^{2}$ & $\mathrm{~g} / \mathrm{L} \mathrm{m}^{2}$ & $\mathrm{~g} / \mathrm{Lm}^{2}$ & $\mathrm{~g} / \mathrm{Lm}^{2}$ & $\begin{array}{c}\text { Plastic } \\
\text { cover }\end{array}$ \\
\hline 1 & F1 & 1.67 & 0 & 0 & 0 & NO \\
\hline 2 & F2 & 1.67 & 5.00 & 0.850 & 0.850 & NO \\
\hline 3 & F3 & 1.67 & 2.33 & 0.397 & 0.397 & NO \\
\hline 4 & F4 & 1.67 & 5.00 & 0.850 & 0.850 & YES \\
\hline 5 & F5 & 1.67 & 2.33 & 0.397 & 0.397 & YES \\
\hline 6 & F6 & 1.67 & 0 & 0 & 0 & YES \\
\hline
\end{tabular}

\section{Experimental research}

Experiments were performed to investigate transient water and $\mathrm{N}$ transport in the soil profile, during and after the fertilization and to compare differences in nitrogen $\mathrm{N}-\mathrm{NO}_{3}{ }^{-}$and $\mathrm{N}-\mathrm{NH}_{4}{ }^{+}$concentrations due to different agricultural practices.

Distributed soil samples were taken in each field from 0 to $44 \mathrm{~cm}$ depth. Six scenarios (table 1) were comparatively analysed. Lettuce growth, $\mathrm{N}-\mathrm{NO}_{3}{ }^{-}$and total nitrogen (Ntotal) accumulation in leaves were studied in each case. 


\subsection{Soil properties measurement}

Soils absorb and retain water, which may be withdrawn by plants during periods between rainfall and irrigations. Water holding capacity refers to the amount of water held between field capacity (at the moist end) and wilting point (at the dry end). Plant available water is that portion of the water holding capacity that can be absorbed by plant and is generally considered to be 50 percent of the water holding capacity. This plant-available water depends on soil properties.

We measured the soil samples total volume $-V_{t}$, pores water volume $-V_{w}$, soil particles volume $-V_{s}$, dry weight of the sample $-m_{s}$, and $m_{w}$ - the mass of water. We calculated: bulk density $-\rho_{b}$ at different depth, grain density $-\rho_{s}$, volumetric water content at saturation, $-\theta_{s}(\%)$, gravimetric water content at saturation $-\omega_{s}(\%)$, volumetric water content in different fields (spatial and temporal variation) $-\theta(z, t)(\%)$, gravimetric water content in different fields (spatial and temporal variation) $-\omega(z, t)(\%)$. The water pressure in the pores of the unsaturated soil was measured using tensiometric tubes and an electronic pressure transducer SMS2500S (fig. 4). The electronic device provides directly the negative relative air pressure $\left(p_{\text {air }}\right)$ inside the top of tensiometric tube. If $d$ is the height of the water column above the soil surface and $\rho_{w}$ the water density, the pressure (suction) in $\mathrm{N} / \mathrm{m}^{2}$, at a depth $z$ below soil surface, is given by:

$$
p=p_{\text {air }}+\rho_{w} g(z+d),
$$

while the same relative pressure $p$ expressed in $\mathrm{cm} \mathrm{H}_{2} \mathrm{O}$ is:

$$
h=\left(p_{\text {air }} / \rho_{w} g\right)+(z+d)<0 .
$$

Suction is usually expressed as:

$$
p F=\log (\operatorname{abs}(h))
$$

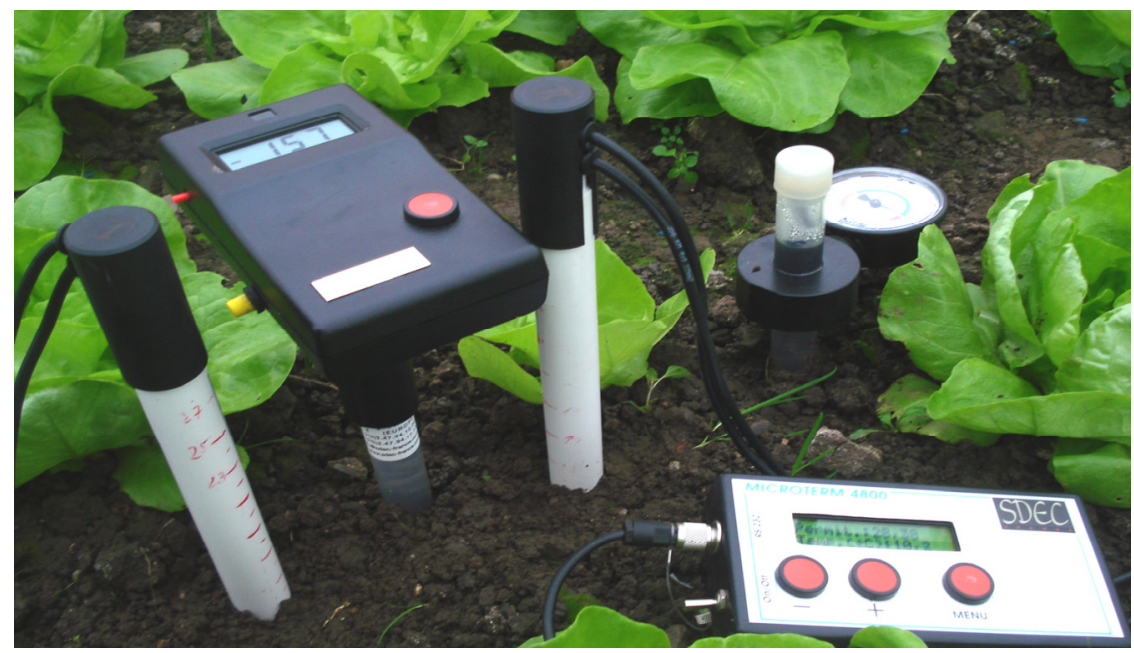

Figure 4: $\quad$ Soil properties measurement. 
The soil volumetric water content was measured using a moisture sensor HMS 9000, coupled to a reading device MICROTERM 4800 (fig. 4). The sensor measures the soil electrical capacity. Between the dielectric soil permittivity $(\varepsilon)$ and its volumetric water content $(\theta)$ a linear relationship:

$$
\varepsilon=A \theta+B
$$

can be expected. The constants A and B have to be calculated for each soil type as part of the system calibration. Volumetric water content $-\theta$, can be read on the display, after a correct calibration of the device.

\subsection{Nitrogen concentration in soil and plant}

The mineral soluble compounds represented by nitrates and ammonia salts are the most mobile form of nitrogen in soil and their quantitative determination is used to characterize the amount of accessible nitrogen in soil.

\subsubsection{Sample preparation}

A mixture formed from 10 grams of soil and $50 \mathrm{~mL}$ of distilled water was stirred for one hour and then filtered off. The resulting solution (extract) was used for quantitative determination of nitrate and ammonium ions. Each sample was analyzed in duplicate and the result was expressed as $\mathrm{mg} / \mathrm{kg}$ fresh weight.

The lettuce leaves were washed with distilled water for dust removal. The samples were sliced and dried on a sheet of paper in order to eliminate the excess of moisture. After these procedures, precisely weighted samples were grounded and treated with acetic acid 2\% according to the ratio 1:20. Colored samples were cleared with animal charcoal. The filtrate was used for nitrate determination (Benton et al. [1]). Each sample was analyzed in duplicate and the result was expressed in $\mathrm{mg} / \mathrm{kg}$ fresh weight.

\subsubsection{Standards and calibration}

All chemicals used were of analytical reagent grade. A $100 \mathrm{~mL}$ standard stock solution containing $100 \mathrm{ppm} \mathrm{N}-\mathrm{NO}_{3}{ }^{-}$was prepared by dissolution in distilled water $0.7219 \mathrm{~g}$ of $\mathrm{KNO}_{3}$, A $100 \mathrm{~mL}$ standard stock solution that contains 100 ppm N-NH${ }_{4}^{+}$was prepared using $0.4715 \mathrm{~g}$ of $\left(\mathrm{NH}_{4}\right)_{2} \mathrm{SO}_{4}$ dissolved in distilled water. Working standards were prepared by dilution of the stock solution. The calibration curves for nitrate and ammonium nitrogen were linear for the studied concentration ranges.

\subsubsection{Analytical methods}

The ammonium nitrogen from water and soil was determined with the spectrophotometric method employing the Nessler reagent (Pansu and Gautheyrou [5]). This method is based on the formation of a yellow-orange colored compound (oxidimercurammonium iodide) as a result of reaction between Nessler reagent and ammonium ions from sample. The color intensity of the newly formed compound is proportional with the ammonium nitrogen concentration in the soil.

$$
\mathrm{NH}_{4}^{+}+2\left[\mathrm{HgI}_{4}\right]^{2-}+4 \mathrm{OH}^{-} \rightarrow \mathrm{HgO} \cdot \mathrm{Hg}\left(\mathrm{NH}_{2}\right) \mathrm{I}+7 \mathrm{I}^{-}+3 \mathrm{H}_{2} \mathrm{O}
$$


The nitrate nitrogen dosage from soil, water and plant were measured with the spectrophotometric method using phenol-2.4-disulphonic acid in basic medium. All the measurements were made at $410 \mathrm{~nm}$ using the VIS spectrophotometer Metertek SP830 Plus. The resulting yellow colored compounds are called nitroderivates. These have absorption maxima at $410 \mathrm{~nm}$ and their color intensity is proportional with the sample nitrates concentrations.

\subsubsection{Total nitrogen - the Kjeldahl method}

The Kjeldahl procedure can be divided into three main steps, as follows: digestion, distillation and titration. In the first step, organic nitrogen is transformed into ammonium sulphate by heating the sample with $\mathrm{H}_{2} \mathrm{SO}_{4}$. For this step, potassium sulphate and copper sulphate are used as catalysts to increase the boiling point of the solution. The chemical decomposition is complete when the solution becomes clear and colorless. The digested sample is alkalinized with $\mathrm{NaOH}$ and the nitrogen is distilled off as ammonia trapped in a boric acid solution. The amount of ammonia nitrogen is then quantified by titration with a standard $\mathrm{H}_{2} \mathrm{SO}_{4}$ solution. A reagent blank is carried through the analysis and the volume consumed for this blank is subtracted from each determination.

\section{Experimental results}

\subsection{Physical characteristics and soil pollution}

Using the measured values: $V_{t}, V_{w}, V_{s}, m_{s}$, and $m_{w}$ (defined in section 3.1), we calculated in table 2: $\rho_{b}\left(\mathrm{~g} / \mathrm{cm}^{3}\right)$ at different depth, $\rho_{s}\left(\mathrm{~g} / \mathrm{cm}^{3}\right), \theta_{s}(\%)$, and $\omega_{s}(\%)$.

Table 2: $\quad$ Soil properties in the experimental field.

\begin{tabular}{|c|c|c|c|c|}
\hline Properties & bulk density & $\begin{array}{c}\text { grain } \\
\text { density }\end{array}$ & $\begin{array}{c}\text { volumetric water } \\
\text { content at } \\
\text { saturation }\end{array}$ & $\begin{array}{c}\text { gravimetric } \\
\text { water content } \\
\text { at saturation }\end{array}$ \\
\hline Definition & $\rho_{b}=\frac{m_{s}}{V_{t}}$ & $\rho_{s}=\frac{m_{s}}{V_{s}}$ & $\theta_{s}(\%)=\frac{V_{w}}{V_{t}} 100$ & $\omega_{s}=\frac{m_{w}}{m_{s}} 100$ \\
\hline Values & $\begin{array}{c}1.25-1.5 \\
\mathrm{~g} / \mathrm{cm}^{3}\end{array}$ & $2.47 \mathrm{~g} / \mathrm{cm}^{3}$ & 50.8 & 39 \\
\hline
\end{tabular}

The soil pressure and moisture content variation in the soil profile (fig. 5) show the influence of irrigation on the water content and water velocity in the soil. The irrigations performed on 24.11.2009 and on 04.12.2010 produce an increase of humidity in the soil profile. Salads roots develop in the top $15 \mathrm{~cm}$, this is where the plants take their water from. Because of plant water uptake and gravitational flow, humidity increases deeper in the soil, with smallest values above $15 \mathrm{~cm}$, and larger values (close to saturation) between 15 and $40 \mathrm{~cm}$. 
Air humidity is very large in the tunnel and the large day-night variations produce water vapor condensation and insure humidity close to the field capacity even between irrigations.
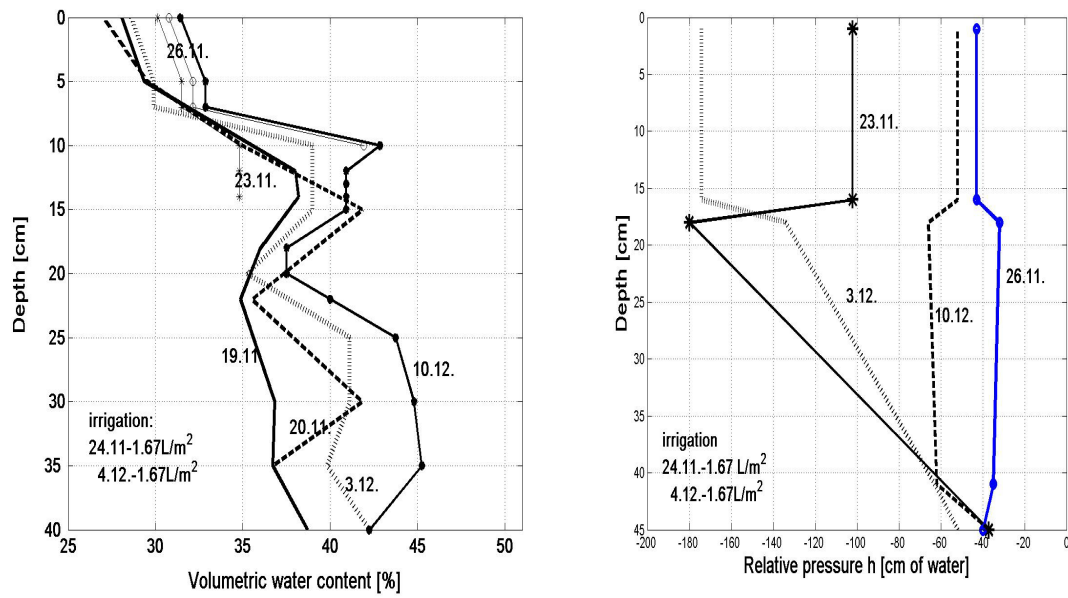

Figure 5: Volumetric water content and suction in the soil profile during the experiment.
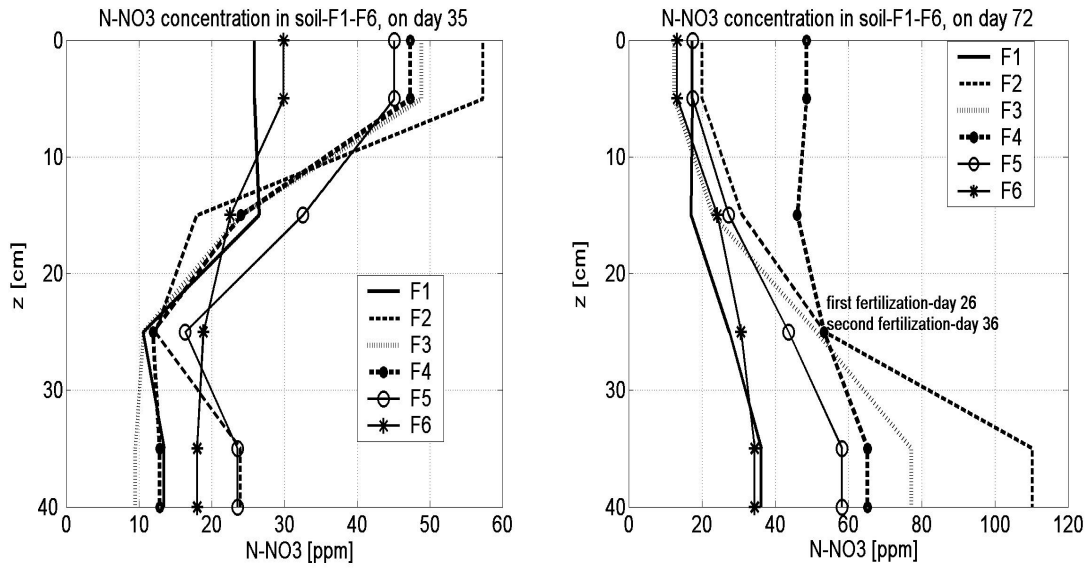

Figure 6: The $\mathrm{N}_{-} \mathrm{NO}_{3}$ concentration in soil profile, in fields $\mathrm{F} 1, \mathrm{~F} 2, . . \mathrm{F} 6$, on day 35 and on day 72 .

Fig. 6 shows the nitrate distribution in soils on day 35 ( 35 days after planting the salad and right before the second fertilization) and on day 72 (36 days after the second fertilization and 20 days after the plants were collected). On day 72 nitrate reaches deeper in the soil (compared to day 35), achieving maximum values between $30-40 \mathrm{~cm}$ depth. Maximum nitrogen fertilization and no plastic 
cover results in maximum nitrate at depth for field F2, while no nitrogen fertilization and plastic cover results in a minimum concentration for field F6.

\subsection{Plant growth description}

During the plant life we measured the number and dimensions of plant leaves, plant diameter, height and volume, and root length and we tried to correlate the plant growth with the nitrogen concentration in leaves, roots, and soil.

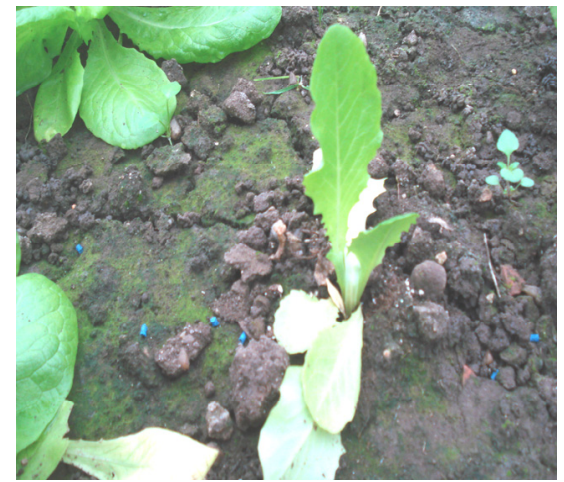

(a)

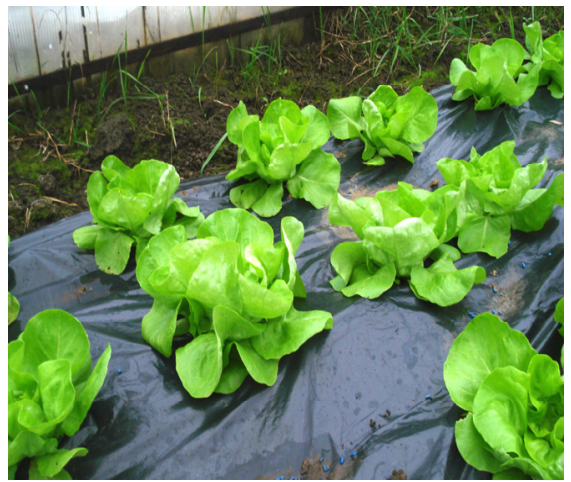

(b)

Figure 7: $\quad$ Lettuce growth level. (a) Typical plant on the first day. (b) Fifth week in field F6 with mulch

To analyze the acquired data we plotted $\mathrm{N}-\mathrm{NO}_{3}{ }^{-}$(fig. 8) and total nitrogen concentration in plant (fig. 9) during its lifetime, $\mathrm{N}-\mathrm{NO}_{3}{ }^{-}$and total nitrogen concentration in the soil profile, for the six fields. To compare the behavior of the plants in the six agronomical conditions and the fertilization method's impact on soil pollution we define a set of parameters which describe the nitrogen accumulation in plant and in the soil profile, and the fertilization efficiency.

We calculate the $\mathrm{N}-\mathrm{NO}_{3}^{-}$and total nitrogen accumulation rate in plant's leaves, in a $\Delta t$ time interval between measurements:

$$
v_{N}=\frac{C(t+\Delta \mathrm{t})-C(t)}{\Delta t},
$$

where $C(t)$ and $C(t+\Delta \mathrm{t})$ are the concentrations at the beginning and at the end of each time interval.

We compared $v_{N-N O 3}(t)$ from fig. 8 and $v_{N \text { total }}(t)$, from fig. 9 , with the plant growth rate (the rate of increase of plant mass) in a time interval $\Delta t$, (fig. 9), defined as:

$$
v_{g}=\frac{m(t+\Delta t)-m(t)}{\Delta t}
$$



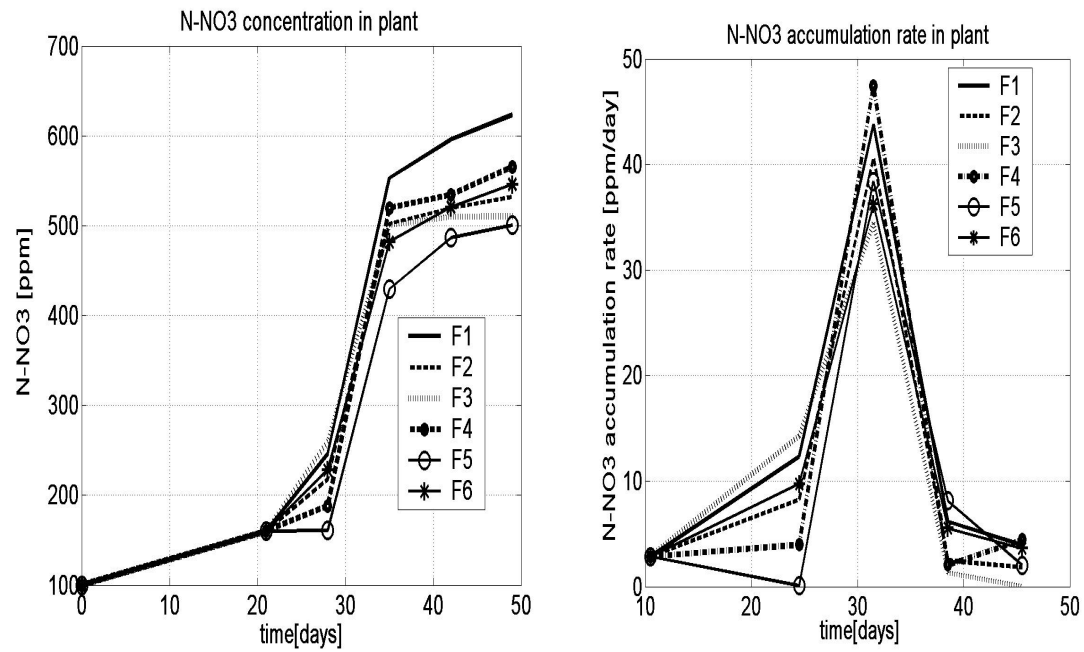

Figure 8: $\quad \mathrm{N}-\mathrm{NO} 3$ concentration in plant and $\mathrm{N}-\mathrm{NO}_{3}$ accumulation rate in plant.
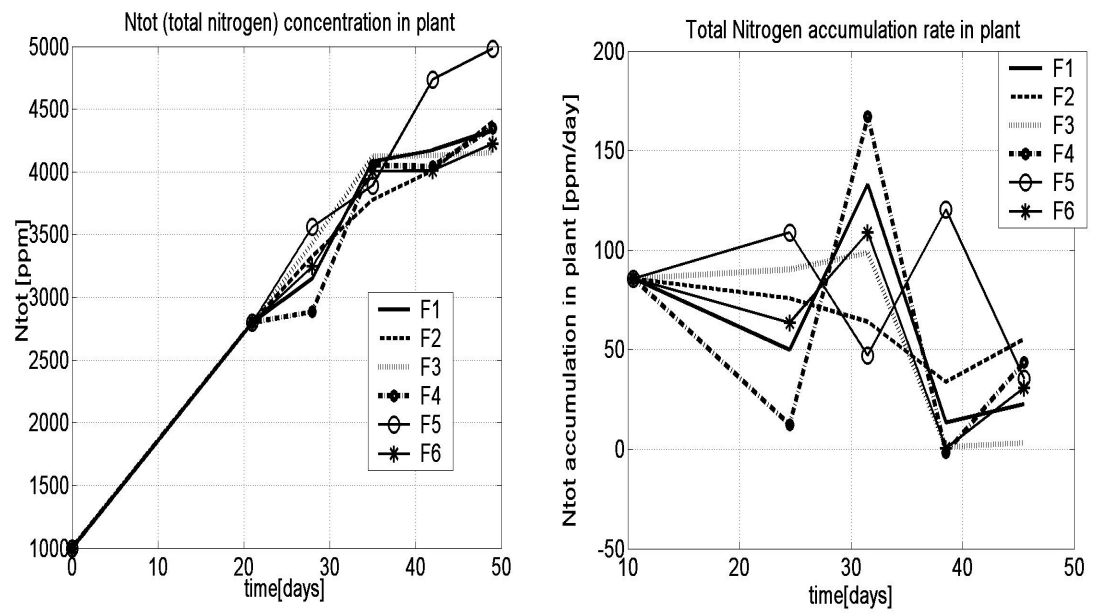

Figure 9: Total nitrogen concentration in plant and total nitrogen accumulation rate in plant.

where $m(t)$ is plant mass at time $t$ and $m(t+\Delta t)$ is plant mass at time $t+\Delta t$. We calculated an efficiency of plant growth in a $\Delta t$ interval of time, like a ratio between the plant growth rate $v_{g}$ and nitrogen accumulation rate $v_{N}$ :

$$
\text { Efficiency of growth }=\frac{v_{g}}{v_{N}}=\frac{m(t+\Delta t)-m(t)}{C_{N-N O 3}(t+\Delta \mathrm{t})-C_{N-N O 3}(t)} .
$$



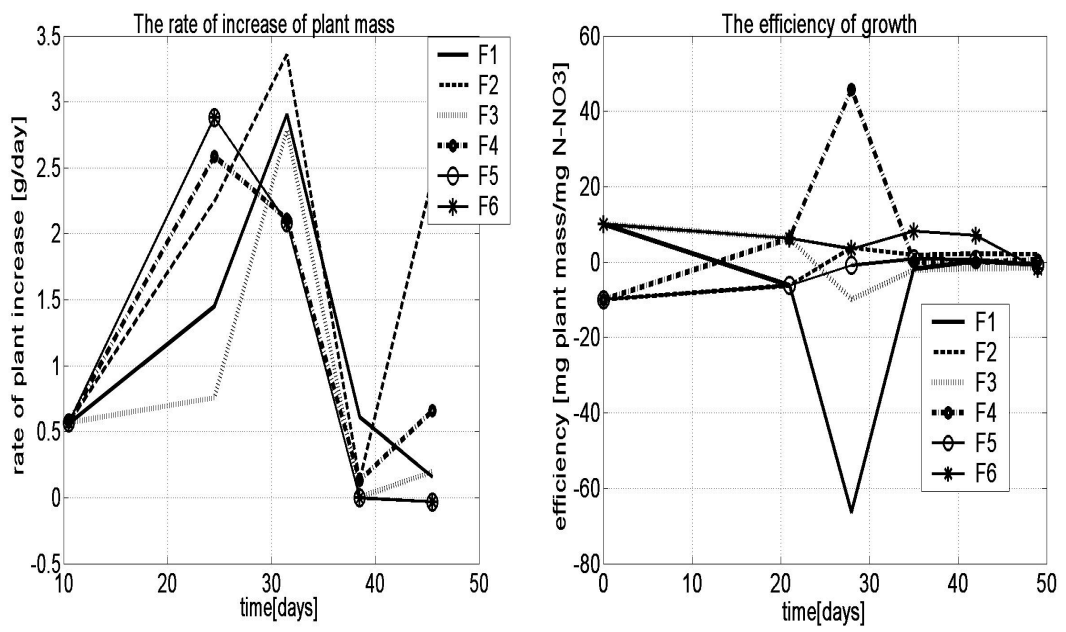

Figure 10: The rate of increase of plant mass and the efficiency of growth.

We studied the time dependence of the efficiency of plant growth in each field (fig. 10).

At every time step $(t)$ we calculate the average plant mass for the 6 fields F1..F6

$$
\bar{m}(t)=\frac{\sum_{j=1}^{6} m(t, j)}{6},
$$

where $m(t, j)$ is the leaf mass for a representative lettuce in field $j$ on day $t$.

The average mass of $\mathrm{N}-\mathrm{NO}_{3}$ for the six fields is:

$$
\bar{m} N O_{3}(t)=\frac{\sum_{j=1}^{6} m N O_{3}(t, j)}{6},
$$

where $\mathrm{mNO}_{3}(t, j)$ is the mass of $\mathrm{N}-\mathrm{NO}_{3}$ contained in a typical lettuce in field $j$.

We defined total plant growth sensitivities to changes in nitrate or changes in total nitrogen, in the field $j, T P G S(j)$ [g of plant/mg N] as:

$$
\begin{aligned}
T P G S_{N-N O 3}(j) & =\frac{m(50, j)-m(1, j)}{m N O 3(50, j)-m N O 3(1, j)}, \\
T P G S_{N \text { tot }}(j) & =\frac{m(50, j)-m(1, j)}{m N \operatorname{tot}(50, j)-m N \operatorname{tot}(1, j)}
\end{aligned}
$$

where $m(50, j)$ and $m(1, j)$ are the plant mass at the end ( $t=50$ days) and at the beginning of the experiment ( $t=1$ day), respectively, $m N O 3(50, j)$ and $m N$ tot $(50, j)$ are the mass of $\mathrm{N}-\mathrm{NO}_{3}$ and total nitrogen contained in lettuce leaves at the end of experiment. Ideally we want these plant growth sensitivities to be large, 
i.e., we want an agricultural practice in which the plant growth is most sensitive to the addition of fertilizer.

To quantify the best fertilization method among the six fields depicted in table 1, we generate a Score matrix, considering four important criteria for a good fertilization method:

1. $T P G S_{N-N O 3}(j)$ needs to be large as explained above;

2. TPGS $S_{N \text { tot }}(j)$ needs to be large;

3. The concentration of $\mathrm{N}^{-\mathrm{NO}_{3}}{ }^{-}$[ppm] in the first $15 \mathrm{~cm}$ under the soil surface (the roots zone) needs to be large at the end of experiment. This ensures that enough nitrate is left in the soil to be useful for future crops.

4. The concentration of $\mathrm{N}-\mathrm{NO}_{3}{ }^{-}$[ppm] between $15 \mathrm{~cm}$ and $40 \mathrm{~cm}$ depth needs to be small at the end of experiment to avoid groundwater pollution.

Each of the six fertilization methods is given a score which reflects how well they accomplish the above criteria ( 1 for the best method and 6 for the worst). The winning field and the best fertilization solution will be the one with the minimum total score.

According to our score matrix, the winning field was field F4, suggesting that the best management practice is to use mulch and two fertilizations.

Table 3: $\quad$ Score matrix.

\begin{tabular}{|c|c|c|c|c|c|c|c|c|}
\hline Criterium & U.M & $\begin{array}{c}\text { Con } \\
\text { d. }\end{array}$ & F1 & F2 & F3 & F4 & F5 & F6 \\
\hline$T P G S_{N-N O 3}(j)$ & $\mathrm{g} / \mathrm{mg} \mathrm{N}$ & Max & 1.52 & 2.19 & 1.21 & 1.60 & 1.49 & 1.48 \\
\hline Score & - & - & 3 & 1 & 6 & 2 & 4 & 5 \\
\hline$T P G S_{\text {Ntot }}(j)$ & $\mathrm{g} / \mathrm{mg} \mathrm{N}$ & $\mathrm{Max}$ & 0.22 & 0.32 & 0.18 & 0.23 & 0.21 & 0.21 \\
\hline Score & - & & 3 & 1 & 6 & 2 & 4 & 5 \\
\hline${\mathrm{N}-\mathrm{NO}_{3}-0-15 \mathrm{~cm}}^{\text {Score }}$ & $\mathrm{ppm}$ & $\mathrm{Max}$ & 36.2 & 110.3 & 77.2 & 65.3 & 58.3 & 34.5 \\
\hline${\mathrm{N}-\mathrm{NO}_{3}-15-40 \mathrm{~cm}}^{\text {Score }}$ & $\mathrm{ppm}$ & $\mathrm{Min}$ & 17.4 & 20.1 & 12.5 & 48.6 & 17.5 & 13.3 \\
\hline Total Score & - & - & 4 & 2 & 6 & 1 & 3 & 5 \\
\hline
\end{tabular}

\section{Conclusions}

Our study quantifies the impact of nitrate fertilization on plant growth and on the transport of nitrate in soil. In particular, we studied the development of plants and the accumulation of $\mathrm{N}^{-\mathrm{NO}_{3}}{ }^{-}$and total nitrogen (Ntotal) in plant leaves for fifty days, in six different fields in which we applied different nutrient fertilization methods. The following ideas emerge from our experimental results:

- the growth rate of the plants which are not covered by plastic (fields F1, F2, F3) depend directly on daytime light intensity and temperature (fig. 2). For fields F1, F2 and F3 the growth rate of plants (fig. 10) reaches its maximum on day 32 
(6 days after the first fertilization), corresponding to the peak in light intensity and temperature. By contrast, covering the field with mulch decorrelates the growth rate from these physical parameters. Maximum growth rate in fields F4, F5 and F6 therefore does not coincide with the maximum of light;

- the second fertilization ensures higher growth rate in field F2 compared to F3 and F4 (fig 10). The lack of a plastic cover and thus more light results in higher growth rates for field F2 compared to F4. By contrast, $v_{g}$ decreases after day 32 and remains low in fields F1 and F6 in which we did not add fertilizer;

- the highest efficiency of plant growth (eqn. (6)) is observed in field F4 on day 27, after the first fertilization. After 35 days the maximum efficiency is in field F6, showing clearly the advantage of covering the field with mulch. After day 49 the efficiency became greater in F2 but the nitrate concentration at $30-40 \mathrm{~cm}$ depth in this field is the biggest compared with the other field as shown in fig. 6;

- initial nitrate in soil is actively used by plants until day 26 , the day of the first fertilization. As expected from previous research, Draghici [2], the plastic cover ensures a maximal efficiency of initial nitrate utilization for fields F4, F5, F6 (fig. 10).

As a result of score matrix analysis, the mulch method with maximum nitrate fertilization, corresponding to field F4, emerges as the best method for nitrogen fertilization of a salad crop. This method insures a minimal nitrate concentration in the plant, the lowest nitrate concentration in the soil below $15 \mathrm{~cm}$, and a large nitrate concentration left in the top $15 \mathrm{~cm}$ of soil, to be used by potential future crops.

\section{Acknowledgements}

This research was developed in the frame of a Romanian National Research Program IDEI, grant 189/2007, sponsored by CNCSIS, Romania.

\section{References}

[1] Benton, J., Jones, J., Wolf, B., Mills, H., Plant Analysis Handbook-A Practical Sampling, Preparation, Analysis, and Interpretation Guide, MicroMacro Publishing, 1991.

[2] Draghici, E.M. The relation between the pot size and some quality index at lettuce nurseling, Lucrari stiintifice USAMVB Horticultura, seria B LI, pp. 68-71, 2008.

[3] Marinov, A.M., Diminescu, M.A., Experimental research and mathematical modelling of soil and groundwater contamination with nitrogen compounds, Proc. of the IX-th. Int. Conf. on Water pollution, eds. C.A. Brebbia, WIT Transactions, UK, pp.115-126, 2008.

[4] Pereira, L.S. \& Quelhas dos Santos, J., Fertilizer and water application, and control of nitrate pollution. Management issues, NATO Advanced Research Workshop on Nitrate Contamination: Exposure, Consequences, and Control, Lincoln, Nebraska, USA, pp.141-162, 1990.

[5] Pansu, M., Gautheyrou, J., Handbook of Soil Analysis (Mineralogical, Organic and Inorganic Methods), Springer - Verlag, Berlin, Heidelberg, 2006. 